\title{
Hydralazine-Induced Dual Antineutrophil Cytoplasmic Antibody Positive Vasculitis and Nephritis
}

\author{
Sandeep A. Padala ${ }^{1}$, Vidya M. Medepalli ${ }^{2}$, Azeem Mohammed ${ }^{1}$, Stanley Nahman ${ }^{1}$ \\ 1. Nephrology, Augusta University Medical College of Georgia, Augusta, USA 2. Medicine, Augusta University Medical \\ College of Georgia, Augusta, USA
}

Corresponding author: Sandeep A. Padala, drspadala@gmail.com

\begin{abstract}
Drug-induced autoimmunity occurs when exposure to a causative agent leads to serologic or clinical autoimmune responses. Syndromes that may be associated with drug-induced autoimmunity include antineutrophil cytoplasmic antibody (ANCA) associated vasculitis (AAV) and drug-induced lupus (DIL). When drug-induced autoimmunity involves the kidney, histological patterns of injury include pauci-immune glomerulonephritis (GN), which occurs with AAV, and immune complex-mediated GN, which is associated with DIL. We present a case of hydralazine-induced dual ANCA-positive vasculitis and nephritis.
\end{abstract}

Categories: Internal Medicine, Nephrology, Rheumatology

Keywords: hydralazine, drug-induced lupus (dil), antineutrophil cytoplasmic antibody (anca), antineutrophil cytoplasmic antibody (anca) associated vasculitis (aav), pauci-immune glomerulonephritis (gn), acute kidney injury (aki), dual anca aav and dil, hydralazine induced dual aav and dil

\section{Introduction}

Hydralazine, an anti-hypertensive arterial vasodilator, was one of the first and remains a common cause of autoimmunity. In cases of hydralazine-associated autoimmune disease, renal involvement is more common with antineutrophil cytoplasmic antibody (ANCA) associated vasculitis (AAV). Drug-induced lupus (DIL) rarely involves the kidney, but the two syndromes may have similar clinical presentations, making an assessment for ANCA important in the evaluation [1,2]. The presence or absence of ANCAs is one of the most helpful clues; ANCAs are positive with AAV and generally not seen with immune complex glomerulonephritis (GN)/DIL [3]. Herein, we present a unique case of DIL nephritis with serologic and histologic features of both diseases.

\section{Case Presentation}

Received 06/12/2020 Review began 06/25/2020 Review ended 06/25/2020 Published 06/29/2020

\section{(c) Copyright 2020}

Padala et al. This is an open access article distributed under the terms of the Creative Commons Attribution License CC-BY 4.0., which permits unrestricted use, distribution, and reproduction in any medium, provided the original author and source are credited.
A 76-year-old Caucasian female was transferred to our institution for the evaluation of non-oliguric acute kidney injury (AKI) on chronic kidney disease (CKD). Her medical history included hypertension, type 2 diabetes mellitus, stage 3 CKD due to diabetic nephropathy (baseline serum creatinine of $1.5-2.0 \mathrm{mg} / \mathrm{dL}$ ), coronary artery disease status post-multiple stents, peripheral arterial disease, and chronic diastolic heart failure. She denied any history of autoimmune disease or alopecia, photosensitive rash, oral ulcers or paresthesias, or family history of autoimmune disease. Home medications included amlodipine $5 \mathrm{mg}$ daily, atenolol $50 \mathrm{mg}$ daily, hydralazine $100 \mathrm{mg}$ every eight hours, isosorbide mononitrate $60 \mathrm{mg}$ daily, losartan 100 $\mathrm{mg}$ daily, aspirin $81 \mathrm{mg}$ daily, clopidogrel $75 \mathrm{mg}$ daily, and atorvastatin $10 \mathrm{mg}$ daily. The patient was initially admitted to the outside facility for acute hypoxemic respiratory failure and a urinary infection, which was treated with ceftriaxone. There was a two-month history of fatigue, arthralgias, and recurrent sinus infections. A CT angiogram ruled out pulmonary embolism, but the serum creatinine rose from 2.0 to 5.2 $\mathrm{mg} / \mathrm{dL}$ within 24 hours. The etiology of the AKI was thought to be contrast nephropathy. Despite supportive care, the renal function worsened and she was transferred to our institution for further evaluation.

Upon admission, vitals included a temperature of $37.4^{\circ} \mathrm{C}$, heart rate of 69 beats per minute, blood pressure of $172 / 69 \mathrm{mmHg}$, respiratory rate of 18 breaths per minute, and $\mathrm{O}_{2}$ saturation of $99 \%$ on room air. On physical examination, she was comfortable, with moist oral mucosa. The lungs were clear to auscultation, and the cardiovascular examination revealed a systolic murmur without jugular venous distension. There was $1+$ bilateral lower extremity edema. There were no rashes or other skin lesions. Nephrology was consulted for non-oliguric AKI with worsening renal indices. Urine microscopy showed many red blood cells but no acanthocytes or casts. Renal ultrasound revealed 12-cm kidneys bilaterally with cortical thinning without mass or obstruction. The initial working diagnosis was AKI on CKD secondary to contrast-induced nephropathy or acute interstitial nephritis following ceftriaxone exposure, atheroembolic disease, and systemic vasculitis. As shown in Table 1, there was an increase in antinuclear antibody (ANA), elevated double-stranded DNA (ds-DNA), ANCAs, and a mildly depressed C3, raising the concern for possible hydralazine-induced AAV and DIL. Hydralazine was stopped, and high-dose steroids were initiated pending a renal biopsy. Due to volume overload and worsening renal indices, renal replacement therapy was also 


\section{Cureus}

initiated. 


\section{Cureus}

\begin{tabular}{|c|c|}
\hline White blood count $\left(4.5-11 \times 10^{3}\right.$ cells $\left./ \mathrm{mm}^{3}\right)$ & 5.1 \\
\hline Red blood count (4.2-5.5 million/mm³) & 2.5 \\
\hline Hemoglobin (12-16 g/dL) & 7.2 \\
\hline Hematocrit (37-47\%) & 21.1 \\
\hline Platelet $\left(150,000\right.$ to $\left.400,000 / \mathrm{mm}^{3}\right)$ & 206 \\
\hline Sodium (135-145 mEq/L) & 135 \\
\hline Potassium (3.5-5.5 mEq/L) & 4.3 \\
\hline Chloride (99-109 mEq/L) & 102 \\
\hline Bicarbonate (20-31 mEq/L) & 24 \\
\hline Blood urea nitrogen (9-23 mg/dL) & 41 \\
\hline Creatinine $(0.6-1.6 \mathrm{mg} / \mathrm{dL})$ & 5.22 \\
\hline Glucose (74-106 mg/dL) & 114 \\
\hline Calcium (8.7-10.4 mg/dL) & 9.0 \\
\hline Albumin (3.2-4.8 g/dL) & 3.2 \\
\hline Total bilirubin (0.3-1.2 mg/dL) & 0.4 \\
\hline Phosphorus (2.4-5.1 mg/dL) & 5.1 \\
\hline Magnesium (1.3-2.7 mg/dL) & 2.0 \\
\hline CRP $(0-0.5 \mathrm{mg} / \mathrm{dL})$ & 3.125 \\
\hline ANA screen & Positive \\
\hline ANA titer & $\geq 1: 640$ \\
\hline $\mathrm{RF}(0-14 \mathrm{IU} / \mathrm{mL})$ & 24 \\
\hline C3 (90-170 mg/dL) & 77 \\
\hline C4 (12-36 mg/dL) & 12 \\
\hline Cryoglobulin & Negative \\
\hline Hepatitis screen & Negative \\
\hline HIV & Negative \\
\hline dsDNA $(<30 \mathrm{IU} / \mathrm{mL})$ & 115 \\
\hline ANCA PR3 antibody $(<0.4 \mathrm{U})$ & 3.5 \\
\hline ANCA MPO antibody (<0.4 U) & 7.6 \\
\hline Anti-histone antibodies $(<1 \mathrm{U})$ & 5.3 \\
\hline SPEP & No monoclonal immunoglobulins detected \\
\hline SIFE & No monoclonal immunoglobulins detected \\
\hline SFLC ratio $(0.26-1.65)$ & 0.7 \\
\hline
\end{tabular}

\section{TABLE 1: Laboratory Data}

CRP, C reactive antibody; ANA, antinuclear antibodies; RF, rheumatoid factor; C3, complement component 3; C4, complement component 4; HIV, human immunodeficiency virus; dsDNA, double-stranded DNA antibody; ANCA PR3, antineutrophil cytoplasmic antibody proteinase 3; ANCA MPO, antineutrophil cytoplasmic antibody myeloperoxidase; SPEP, serum protein electrophoresis; SIFE, serum immunofixation; SFLC ratio, serum free light chains ratio (Kappa/Lambda) 


\section{Cureus}

The biopsy (Figures 1, 2) revealed immunoglobulin (Ig) M dominant immune complex-mediated focal proliferative GN. Light microscopy showed one segmental crescent, mild mesangial hypercellularity, acute tubular injury, interstitial edema, foci of mononuclear cells with neutrophilic interstitial infiltration, and red cell casts. Immunofluorescence showed granular mesangial and capillary wall staining for IgM (4+), C3 (4+), lambda light chain (3-4+), trace IgA, and segmental trace IgG. Electron microscopy showed definitive immune complex deposits in the mesangial, para-mesangial, and subendothelial compartments.

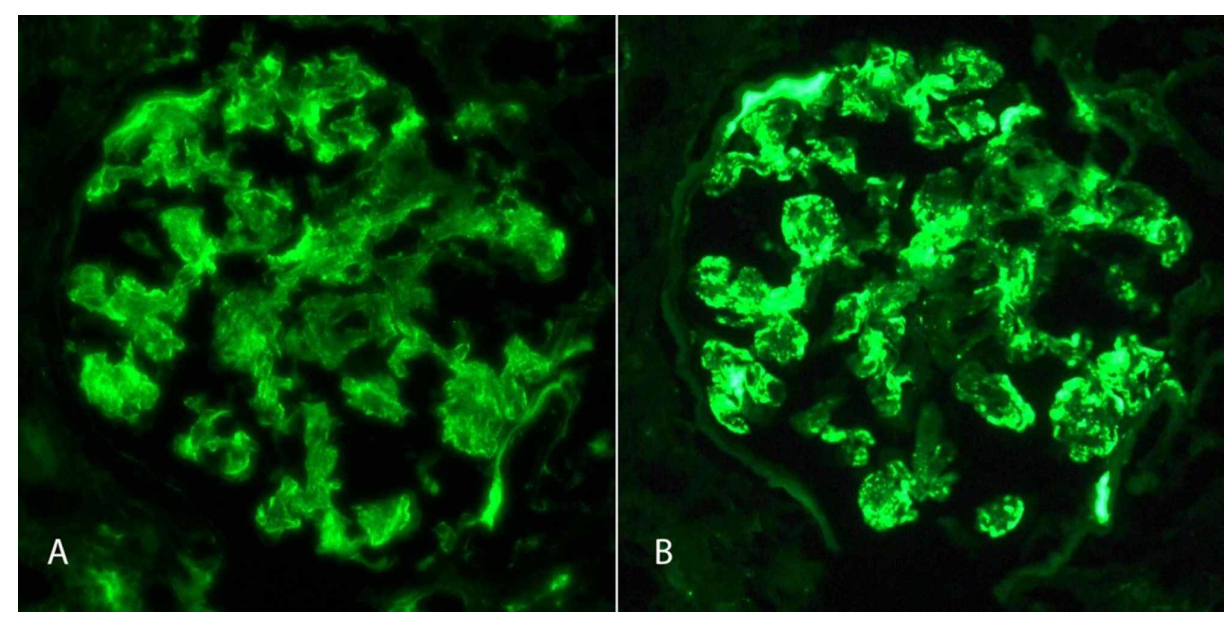

\section{FIGURE 1: Immunofluorescence Microscopy}

Ten glomeruli were available for evaluation, of which none were globally sclerosed. There was granular mesangial and capillary wall staining with antisera specific for IgG (segmental trace), IgA (trace), IgM (4+), complement factor C3 (4+), kappa light chain (1+), and lambda light chain (3-4+). No staining was seen with $\mathrm{C} 1 \mathrm{q}$ and fibrin. No diagnostic extraglomerular staining was seen with antisera specific for lgG.

Ig, immunoglobulin

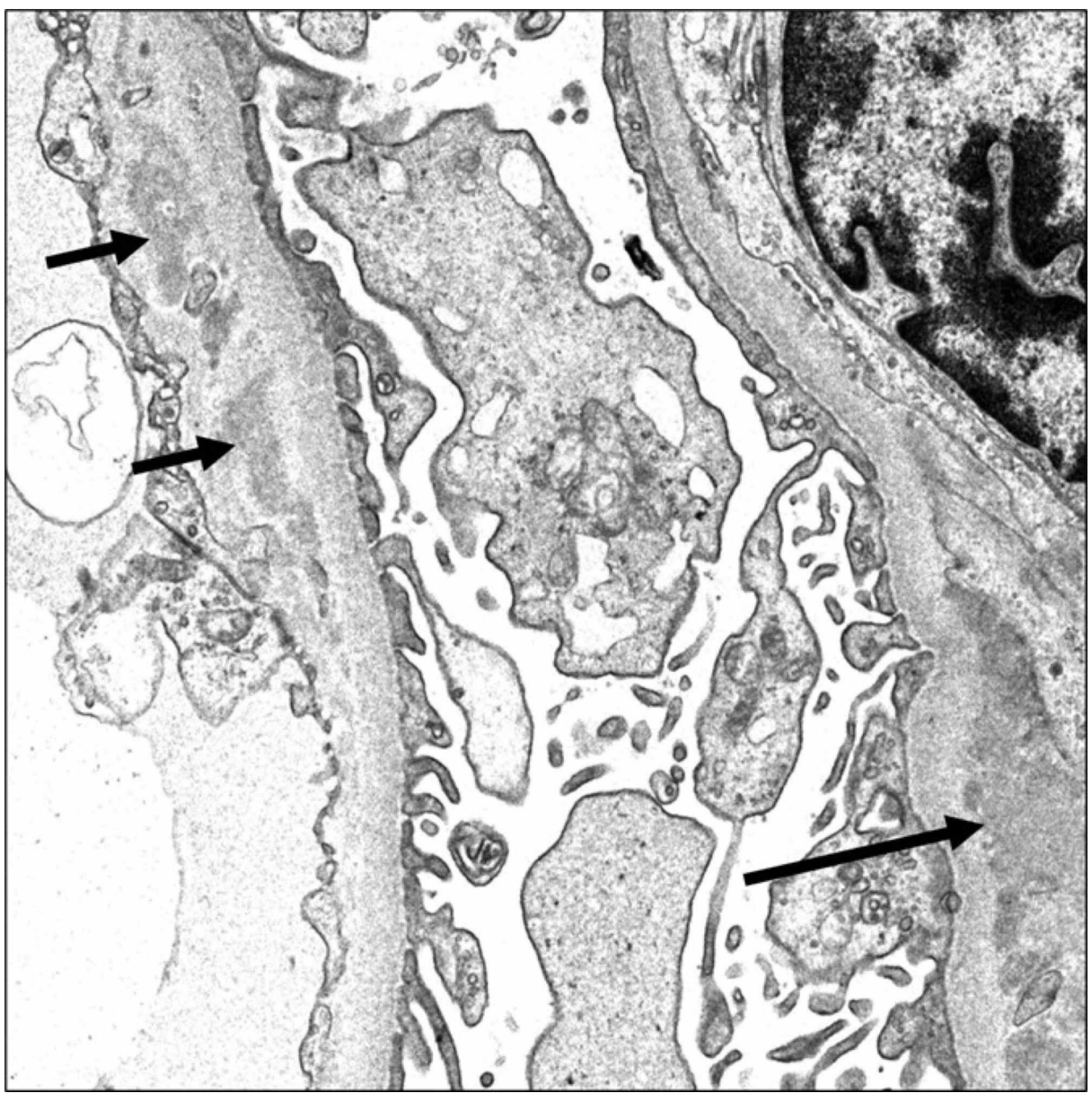




\section{FIGURE 2: Electron Microscopy}

Out of two glomeruli, none revealed global glomerulosclerosis. The glomeruli revealed mild mesangial matrix expansion. The tubulointerstitial compartment revealed mild acute tubular injury. One small interlobular artery cross-section with mild intimal fibroelastosis was also seen. Ultrastructural examination demonstrated peripheral capillary loops segmental thickening of the lamina densa associated with segmental remodeling of some of the capillary loops. There was widespread effacement of the overlying foot processes with associated moderate microvillus transformation of the podocyte cytoplasm. Mesangial, paramesangial, and subendothelial immune complex-type electron-dense deposits were noted. The immune complex-type electron-dense deposits did not reveal any characteristic and diagnostic substructure or organoid pattern. No endothelial cytoplasmic tubuloreticular inclusions were identified.

Light microscopy: Out of three glomeruli per level of section for evaluation, none were globally sclerosed. The glomeruli revealed mild mesangial matrix expansion with associated mild mesangial cells hypercellularity and segmental mild endocapillary hypercellularity. One segmental cellular crescent was noted. No fibrinoid necrosis or segmental scarring was identified. The tubulointerstitial compartment revealed diffuse acute tubular injury, interstitial edema, foci of mononuclear cells with neutrophilic component interstitial infiltration, and red blood cell cast formation. There were mild interstitial fibrosis and tubular atrophy. The few sampled arterioles were unremarkable, and the arteries were not sampled.

Based on the biopsy findings, AKI was presumed to be from hydralazine-induced dual ANCA AAV and DIL. The patient was offered cyclophosphamide or rituximab in addition to continuing prednisone, but she declined an escalation of immunosuppression. The renal function did not recover, and she became dialysisdependent.

\section{Discussion}

Our patient most likely demonstrated AKI from hydralazine-induced dual ANCA AAV and DIL. This hypothesis is supported by the renal biopsy findings and clinical serologies. ANCA AAV and DIL have each been reported with hydralazine, but the present case shows the unusual occurrence of the simultaneous expression of both conditions in the same patient.

Hydralazine is often implicated as a causative factor in DIL, but it rarely causes AAV. DIL is characterized by fever, polyarthralgias, and pleuritic pain [1,2], whereas AAV is more commonly associated with leukocytoclastic vasculitis, pulmonary infiltrates, elevated ANCA levels, and pauci-immune GN on renal biopsy [1,2]. Our patient exhibited no clear clinical signs of DIL or AAV, but the serologic evaluation suggested the possibility of both diagnoses. A renal biopsy confirmed the hypothesis. While the two processes have distinct histopathologic findings, ANCAs are an important means of differentiating the two before biopsy [3]. It has been shown that both DIL and ANCA AAV may represent a spectrum of the same disease [2].

The serologic findings in DIL typically include a positive ANA, anti-histone antibody, low complements $[4,5]$, and variable anti-dsDNA (double-stranded DNA antibody) antibody levels. Renal involvement in DIL is rare, with only a few cases described in the literature. Shapiro et al. described a case of DIL due to hydralazine, with the biopsy demonstrating immune complex-mediated GN [6]. Sheikh et al. reported a case of crescentic GN in a patient with DIL due to procainamide [7].

The serologic findings in drug-induced AAV include a positive ANA, ANCA, and variable anti-dsDNA and anti-histone antibody and complement levels [8]. Renal involvement is a common occurrence in cases of AAV due to hydralazine and is typically seen early in the clinical course. Renal biopsy in these cases demonstrates pauci-immune GN [5,9].

A case series of ANCA-associated GN due to hydralazine by Kumar et al. discusses the overlapping clinicopathological features of DIL and AAV and proposes that hydralazine-associated vasculitis and lupus are related disease processes that exist on a continuum [2]. The risk-benefit ratio and implications of continuing therapy should be considered in the case of a positive or rising ANA titer [2]. Espinosa et al. also described the simultaneous presentation of DIL and ANCA AAV secondary to hydralazine in a sarcoid patient [10]. Our case supports the co-expression of these diseases in patients treated with hydralazine. The presence of end-organ damage implies poor prognosis with high morbidity and mortality [11].

It has been reported that the overlapping presentation of both DIL and ANCA AAV have positive ANA, antihistone antibodies, ds-DNA, elevated ANA, and ANCA titers [11], as evidenced in our case. The presence of anti-histone antibodies in a patient on hydralazine should raise the suspicion of DIL [12,13]. The risk of hydralazine-induced DIL is high in females, slow hepatic acetylators, human leukocyte antigen-DR4 (HLADR4 genotype), doses more than $200 \mathrm{mg} /$ day, and individuals with the null gene for complement system protein C4 [14,15].

When hydralazine is suspected to be the cause, there is a temporal correlation with improvement in the renal indices after the medication is stopped [12]. The possibility of hydralazine-induced vasculitis should be 
suspected in any patient presenting with pulmonary-renal syndromes [3]. Our case is unique since hydralazine-induced lupus nephritis is a rare occurrence, with concurrent AAV causing further renal damage. The most important step in the management of this rare disease is discontinuation of the medication. If there is no improvement and or worsening of the renal indices, then treatment with plasmapheresis, cyclophosphamide, or rituximab should be considered [16]. Our patient was on hydralazine $100 \mathrm{mg}$ every eight hours for more than five years, which could have increased the risk of AAV and DIL.

\section{Conclusions}

Our case represents the overlapping features of both AAV and DIL. Although the patient had a clinical and laboratory presentation consistent with ANCA vasculitis, renal biopsy showed immune complex deposits and was suggestive of DIL nephritis. Hydralazine-associated vasculitis and lupus are related disease processes that exist on a continuum. We would like to point out that since hydralazine is known to cause DIL and ANCA vasculitis, is it imperative to get a baseline ANA titers and monitor the titers periodically if prolonged therapy is indicated, even if the patient is asymptomatic. Further studies regarding monitoring the titers may help us guide in a better direction and avoid the risk of drug reactions. The risk-benefit ratio and implications of continuing therapy should be carefully considered in the case of a positive or rising ANA titer. Increased awareness and a high degree of clinical suspicion can help the clinicians diagnose this rare condition caused by the common medication, thus improving overall morbidity and mortality.

\section{Additional Information}

\section{Disclosures}

Human subjects: Consent was obtained by all participants in this study. Conflicts of interest: In compliance with the ICMJE uniform disclosure form, all authors declare the following: Payment/services info: All authors have declared that no financial support was received from any organization for the submitted work. Financial relationships: All authors have declared that they have no financial relationships at present or within the previous three years with any organizations that might have an interest in the submitted work. Other relationships: All authors have declared that there are no other relationships or activities that could appear to have influenced the submitted work.

\section{References}

1. Cameron HA, Ramsay LE: The lupus syndrome induced by hydralazine: a common complication with low dose treatment. Br Med J (Clin Res Ed). 1984, 289:410-412. 10.1136/bmj.289.6442.410

2. Kumar B, Strouse J, Swee M, Lenert P, Suneja M: Hydralazine-associated vasculitis: overlapping features of drug-induced lupus and vasculitis. Semin Arthritis Rheum. 2018, 48:283-287. 10.1016/j.semarthrit.2018.01.005

3. Dobre M, Wish J, Negrea L: Hydralazine-induced ANCA-positive pauci-immune glomerulonephritis: a case report and literature review. Ren Fail. 2009, 31:745-748. 10.3109/08860220903118590

4. Hogan J, Markowitz G, Radhakrishnan J: Drug-induced glomerular disease: immune-mediated injury. Clin J Am Soc Nephrol. 2015, 10:1300-1310. 10.2215/CJN.01910215

5. Ihle BU, Whitworth JA, Dowling JP, Kincaid-Smith P: Hydralazine and lupus nephritis. Clin Nephrol. 1984, 22:230-238.

6. Shapiro KS, Pinn VW, Harrington JT, Levey AS: Immune complex glomerulonephritis in hydralazineinduced SLE. Am J Kidney Dis. 1984, 3:270-274. 10.1016/s0272-6386(84)80044-X

7. Sheikh TK, Charron RC, Katz A: Renal manifestations of drug-induced systemic lupus erythematosus . Am J Clin Pathol. 1981, 75:755-762. 10.1093/ajcp/75.5.755

8. Yokogawa N, Vivino FB: Hydralazine-induced autoimmune disease: comparison to idiopathic lupus and ANCA-positive vasculitis. Mod Rheumatol. 2009, 19:338-347. 10.1007/s10165-009-0168-y

9. Choi HK, Merkel PA, Walker AM, Niles JL: Drug-associated antineutrophil cytoplasmic antibody-positive vasculitis: prevalence among patients with high titers of antimyeloperoxidase antibodies. Arthritis Rheum. 2000, 43:405-413. 10.1002/1529-0131(200002)43:2<405::AID-ANR22>3.0.CO;2-5

10. Espinosa MC, Ding B, Choi K, Cohen DN, Marcelli M, Ifoeze OW: A simultaneous presentation of druginduced lupus with drug-induced ANCA vasculitis secondary to hydralazine use in a patient with sarcoidosis. Proc (Bayl Univ Med Cent). 2019, 32:231-234. 10.1080/08998280.2019.1570422

11. Almroth G, Eneström S, Hed J, Samuelsson I, Sjöström P: Autoantibodies to leucocyte antigens in hydralazine-associated nephritis. J Intern Med. 1992, 231:37-42. 10.1111/j.1365-2796.1992.tb00496.x

12. Hess E: Drug-induced lupus. N Engl J Med. 1988, 318:1460. 10.1056/NEJM198806023182209

13. Burlingame RW, Rubin RL: Drug-induced anti-histone autoantibodies display two patterns of reactivity with substructures of chromatin. J Clin Invest. 1991, 88:680-690. 10.1172/JCI115353

14. Speirs C, Fielder AH, Chapel H, Davey NJ, Batchelor JR: Complement system protein C4 and susceptibility to hydralazine-induced systemic lupus erythematosus. Lancet. 1989, 1:922-924. 10.1016/s01406736(89)92506-3

15. Batchelor JR, Welsh KI, Tinoco RM, et al.: Hydralazine-induced systemic lupus erythematosus: influence of HLA-DR and sex on susceptibility. Lancet. 1980:1107-1109. 10.1016/s0140-6736(80)91554-8

16. Zuckerman R, Patel M, Costanzo EJ, Dounis H, Haj RA, Seyedali S, Asif A: Hydralazine-associated adverse events: a report of two cases of hydralazine-induced ANCA vasculitis. J Bras Nefrol. 2018, 40:193-197.

10.1590/2175-8239-jbn-3858 